\title{
Association between nonalcoholic fatty liver disease and Helicobacter pylori infection in Dali City, China
}

\author{
Ping Yan, MD, Bocheng Yu, BS, Min Li, MD, Weidong Zhao, PhD.
}

\begin{abstract}
الأهداف : لمعرفة ما إذا كانت عدوى هيليكوباكتر بيلوري (H. pylori)

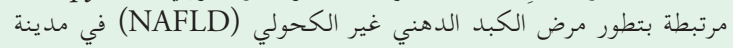
دالي، الصين.

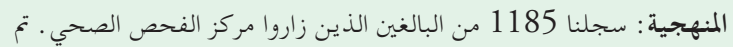

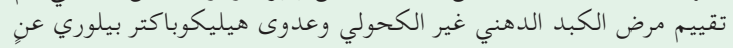

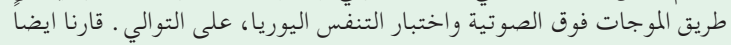

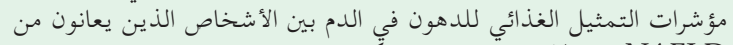
NAFLD

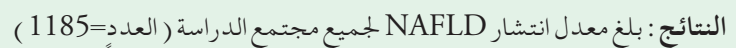

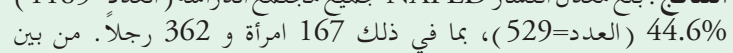
529 شخصًا مصاب $44 \%$ كعامل خطر مهم ومستقل عابل عامل الثقة

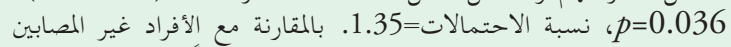

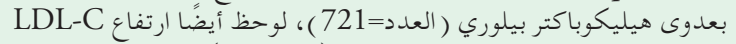

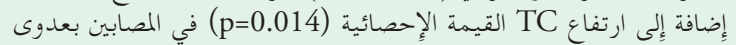

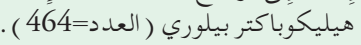

الخلاصة : وجدنا ارتباط إيجابي بين عدوى هيليكوباكتر بيلوري وNAFLD.
\end{abstract}

Objectives: To investigate whether gastric Helicobacter pylori (H. pylori) infection is associated with the progress of nonalcoholic fatty liver disease (NAFLD) in Dali city, China.

Methods: One thousand and one hundred eighty-five adults who had visited a health examination center were enrolled. Nonalcoholic fatty liver disease and H. pylori infection were assessed by abdominal color ultrasound and ${ }^{13} \mathrm{C}$-urea breath test, respectively. Serum lipid metabolic indices were compared between NAFLD subjects without and with $H$. pylori infection.

Results: The prevalence rate of NAFLD for the entire study population $\quad(n=1185)$ was $44.6 \% \quad(n=529)$, including 167 women and 362 men. Among 529 NAFLD subjects, $H$. pylor $i$ was confirmed as a significant and independent risk factor for NAFLD (95\% CI 1.021.79, $p=0.036, \mathrm{OR}=1.35$ ). Compared with $H$. pylori non-infected individuals $(\mathrm{n}=721)$, higher contents of
LDL-C $(p=0.012)$ along with TC $(p=0.014)$ were also observed in $H$. pylori-infected individuals $(\mathrm{n}=464)$.

Conclusion: A positive correlation is identified between H. pylori infection and NAFLD.

Keywords: $H$. pylori infection, nonalcoholic fatty liver disease, lipid metabolic dysfunction, Dali city

Saudi Med J 2021; Vol. 42 (7): 735-741

doi: 10.15537/smj.2021.42.7.20210040

From the Department of Gastroenterology (Yan, Li), from the Department of Clinical Laboratory (Yu, Zhao), the First Affiliated Hospital, Dali University, and from the Department of Hematology (Li), BaoShan People's Hospital, Dali, China.

Received 18th January 2021. Accepted 11th May 2021.

Address correspondence and reprint request to: Dr. Weidong Zhao, Department of Clinical Laboratory, the First Affiliated Hospital, Dali University, Dali, China.E-mail: wdzhao@dali.edu.cn

$\mathrm{N}$ onalcoholic fatty liver disease (NAFLD) is a frequent metabolic condition in humans, creating a global public health concern. ${ }^{1}$ An estimated 400 million people have NAFLD in China, and it has been linked to more than 100 thousand deaths., ${ }^{2,3}$ Research evidence shows that NAFLD is strongly related to insulin resistance, metabolic syndrome, as well as obesity. ${ }^{4}$ However, the association of gastrointestinal microbes to the pathogenesis of NAFLD remains unclear. $^{5}$

Helicobacter pylori (H. pylori) pose a threat to nearly half of the world's population. ${ }^{6,7}$ Infection of $H$. pylori is commonly responsible for gastritis, gastric cancer, as well as peptic ulcer disease. In particular, novel research findings have opined a strong link between $H$. pylori infection and extra-digestive manifestations, namely, diabetes, obesity, liver tumors, cardiovascular disease, and lipid metabolism disorders. ${ }^{8}$ Notably, in recent years, $H$. pylori infection has been viewed as a 
potentially important causative agent associated with the development of NAFLD.

However, connection linking $H$. pylori infection to NAFLD remains debated. Positive associations of NAFLD with $H$. pylori infection have been investigated in numerous studies, ${ }^{9-11}$ whereas some studies have reported conflicting results. ${ }^{12-15}$ Under these circumstance, more clinical data are needed to address the connection of NAFLD with $H$. pylori infection. Locally, no studies regarding NAFLD and $H$. pylori association are available. Therefore, herein, we primarily purposed to explore the association of NAFLD with $H$. pylori infection in medical check-ups in Dali city, a Bai ethnic minority area in western Yunnan province of China.

Methods. We conducted this retrospective research using electronic medical data from participants (age, $>18$ years) who underwent comprehensive health check-ups at a tertiary hospital in Dali city between January 2017 and June 2019 (Figure 1). We included data from participants consisting of anthropometric parameters and results of ${ }^{13} \mathrm{C}$-urea breath test (UBT), serum biochemistry, ultrasound color imaging of the abdomen, and ultrasound color imaging of neck. We excluded subjects who had (i) a history of significant alcohol intake: females ( $\geq 140 \mathrm{~g} /$ week), and males ( $\geq 210 \mathrm{~g} /$ week); (ii) a treatment history with amiodarone, methotrexate, glucocorticoid and tamoxifen; (iii) individuals with celiac disease, Wilson's disease, viral hepatitis, lack of beta lipoprotein, autoimmune hepatitis, total parenteral nutrition, and congenital lipid atrophy; (iv) those under treatment with proton pump inhibitors (PPI) for 2 weeks or under treatment with bismuth salts and antibiotics for 4 weeks prior to the ${ }^{13} \mathrm{C}-\mathrm{UBT}$; (v) individuals with upper gastrointestinal disorders' history; (vi) participants with cancer or mental conditions; and (vii) previously underwent gastric surgery. The ethical committee of Dali University Hospital granted the study protocol and ethical approval (No: 20200118).

Physical examinations and serum biochemical examinations. Anthropometric parameters (weight along with height) were also assessed. And body mass index (BMI) was determined as $\mathrm{kg} / \mathrm{m}^{2}$. We recorded the systolic, as well as diastolic blood pressure during the

Disclosure. Authors have no conflict of interests, and the work was not supported or funded by any drug company. physical examination. A fully automatic biochemical system (Hitachi 7600, Tokyo, Japan) was used to test the serum biochemical indices: alanine aminotransferase (ALT), total cholesterol (TC), aspartate aminotransferase (AST), low-density lipoprotein cholesterol (LDL-C), fasting plasma glucose (FPG), urea, albumin (ALB), triglyceride (TG), high-density lipoprotein cholesterol (HDL-C), creatinine, and uric acid.

Assessment of NAFLD. Each of the participant was subjected to Color Doppler ultrasound imaging of the abdomen by an independent radiologist with a Logiq E8 radiology ultrasound equipment (GE Corp., Chicago, IL, USA). We diagnosed NAFLD on the basis of the results of the ultrasound photographs and the current diagnostic reference criteria. ${ }^{16}$

Establishment of H. pylori infection. Each subject was assessed for $H$. pylori infection using ${ }^{13} \mathrm{C}-\mathrm{UBT}$ kit (Headway, Zhonghe, Shenzhen, China). After $\geq 8$ hours of fasting overnight, we orally administered a $75 \mathrm{mg}$ 13C-urea capsule to all subjects and collected 2 samples of exhaled breath at baseline, as well as 30 mins after the capsule was consumed. We analyzed the samples using a HCBT-01 ${ }^{13} \mathrm{C}$ detection system (Headway, Shenzhen, China). If the results of 2 samples differed by $>4 /$ thousand $(\delta \% 0)$ meant a participant was positive for H. pylori infection, as previously described. ${ }^{17}$

Statisticalstatistics. The characteristics of the subjects are presented as mean $\pm S D$ for normally distributed continuous variables and as percentages for categorical data. We compared the characteristics of subjects across the NAFLD status with the Chi-square test or t-test. Then logistic regression was employed to investigate the effect of various parameters on NAFLD and $H$. pylori infection association by adjusted odds ratios (ORs). We also constructed 4 different models. Model 1 included only $H$. pylori infection. Age and carotid plaque status were added to Model 2. Model 3 was Model 2 with further adjustment for ALT, AST, and UA levels. Finally, more potential indicators and confounders, including FPG, TC, TG, SBP, DBP, LDL-C, and BMI were added to Model 4. Point-biserial correlation was employed to evaluate the correlation between $H$. pylori infection and TC, as well as LDL-C.

Statistical analysis was implemented in the SPSS Statistics 23.0 software (for windows, IBM, Armonk, NY, USA). P-value of $<0.05$ signified statistical significance.

Results. Overall, 1185 participants (407 women and 778 men) were enrolled. The prevalence rate of NAFLD for the entire study population $(\mathrm{n}=1185)$ was $44.6 \%(n=529)$, including 167 women and 362 men. 
Participants with NAFLD were significantly older, with lower levels of HDL-C and higher weight, SBP, and DBP, as well as elevated contents of TC, AST, TG, FPG, LDL-C, and ALT (all $p<0.05$ ). Differences in ALB contents and height between the NAFLD and non-NAFLD subjects were not significant $(p>0.05)$. A higher prevalence rate of $H$. pylori infection was reported in the individuals with NAFLD (43.5\%) relative to that of in the individuals without NAFLD (35.7\%, $p=0.007$ ) (Table 1).

Table 2 summarizes several risk factors related to NAFLD after logistic regression analysis. The levels of ALT (OR $=1.02$, 95\% CI 1.01-1.03), BMI $(\mathrm{OR}=4.38,95 \%$ CI 3.29-5.84), FPG $(\mathrm{OR}=2.29,95 \%$ CI 1.31-3.40), TG (OR $=2.33,95 \%$ CI 1.73-3.14), and $\mathrm{DBP}(\mathrm{OR}=1.73,95 \%$ CI 1.17-2.56) were independent risk factor of NAFLD (all $p<0.05$ ). Notably, H. pylori was similarly a strong independent risk factor for NAFLD (95\% CI 1.02-1.79, OR=1.35, $p=0.036$ ). These findings illustrate that $H$. pylori functions as a mediator of NAFLD.

Furthermore, to clarify the link between NAFLD and $H$. pylori infection, we constructed 4 different models. Consequently, $H$. pylori infection (unadjusted $\mathrm{OR}=1.38$; for $95 \%$ CI 1.09-1.75, $p=0.007$ ) was remarkably linked to NAFLD as indicated in Table 3. Additional adjustment for carotid plaque and age attenuated the result was 95\% CI 1.05-1.68, OR $=1.33, p=0.020$. However, after adjusting the model for AST, UA, and ALT, the OR for NAFLD was escalated (95\% CI 1.10-1.83, OR=1.42, $p=0.008)$. In the fully adjusted model (Model 4), infection of $H$. pylori correlated with an elevated risk of NAFLD (95\% CI 1.02-1.79, OR 1.35, $p=0.036$ ). To elucidate the potential underlying mechanisms of $H$. pylori infection in NAFLD, we conducted an analysis to determine the correlation of $H$. pylori infection with several metabolic syndrome constituents. Compared to the $H$. pylori (-) individuals, the $H$. pylori $(+)$ individuals had elevated TC and LDL-C contents (all $p<0.05$, Table 4). However, the differences in FPG $(p=0.492)$, TG $(p=0.409)$, DBP $(p=0.614)$, BMI $(p=0.142)$, SBP $(p=0.776)$, and HDL-C $(p=0.087)$ between these 2 groups were not significant. Furthermore, point-biserial correlation was employed to evaluate the correlation between $H$. pylori infection and TC, as well as LDL-C.

Table 1 - Comparison of study participants demographics and biochemical profiles between cgroup and non-NAFLD group.

\begin{tabular}{|c|c|c|c|c|}
\hline $\begin{array}{l}\text { Demographics and biochemical } \\
\text { profiles }\end{array}$ & $\begin{array}{l}\text { NAFLD group } \\
(\mathrm{n}=529)\end{array}$ & $\begin{array}{l}\text { Non-NAFLD group } \\
(\mathrm{n}=656)\end{array}$ & $\mathrm{t} / \chi^{2}$ & $P$-value \\
\hline Gender, male, N (\%) & $362(68.4)$ & $416(63.4)$ & 3.268 & 0.071 \\
\hline Helicobacter pylori (+), N (\%) & $230(43.5)$ & $234(35.7)$ & 7.285 & 0.007 \\
\hline Carotid plaque (+), N (\%) & $104(19.7)$ & $63(9.6)$ & 24.461 & 0.000 \\
\hline Age (years) & $43.50 \pm 9.94$ & $40.90 \pm 11.28$ & -4.211 & 0.000 \\
\hline Body mass index $\left(\mathrm{kg} / \mathrm{m}^{2}\right)$ & $26.83 \pm 3.48$ & $23.02 \pm 3.12$ & -19.594 & 0.000 \\
\hline Systolic blood pressure $(\mathrm{mm} \mathrm{Hg})$ & $125.42 \pm 16.81$ & $117.34 \pm 15.56$ & -8.573 & 0.000 \\
\hline Diastolic blood pressure (mm Hg) & $81.75 \pm 11.98$ & $76.12 \pm 10.76$ & -8.421 & 0.000 \\
\hline Total cholesterol $(\mathrm{mmol} / \mathrm{L})$ & $5.36 \pm 1.34$ & $4.98 \pm 0.94$ & -6.276 & 0.000 \\
\hline Triglyceride $(\mathrm{mmol} / \mathrm{L})$ & $2.43 \pm 1.57$ & $1.31 \pm 0.82$ & -11.562 & 0.000 \\
\hline HDL-C (mmol/L) & $1.22 \pm 0.34$ & $1.44 \pm 0.42$ & 9.866 & 0.000 \\
\hline LDL-C (mmol/L) & $3.15 \pm 0.89$ & $2.92 \pm 0.78$ & -4.490 & 0.000 \\
\hline Fasting plasma glucose $(\mathrm{mmol} / \mathrm{L})$ & $5.19 \pm 1.62$ & $4.69 \pm 1.20$ & -6.016 & 0.000 \\
\hline Alanine aminotransferase (U/L) & $32.50 \pm 9.12$ & $20.82 \pm 10.91$ & -11.810 & 0.000 \\
\hline Aspartate aminotransferase (U/L) & $26.15 \pm 6.28$ & $20.40 \pm 4.14$ & -5.107 & 0.000 \\
\hline Albumin $(\mathrm{g} / \mathrm{L})$ & $47.58 \pm 3.11$ & $47.26 \pm 3.61$ & -1.573 & 0.116 \\
\hline
\end{tabular}

LDL-C: low-density lipoprotein cholesterol, HDL-C: high-density lipoprotein cholesterol 
Table 2 - Univariate regression analysis for risk factors associated with nonalcoholic fatty liver disease (NAFLD).

\begin{tabular}{lcccccc}
\hline Risk Factors & $\beta$ & S.E. & Wald & P-value & Odds ratio & $\begin{array}{c}95 \% \\
\text { Confidence intervals }\end{array}$ \\
\hline Helicobacter pylori & 0.30 & 0.14 & 4.38 & 0.036 & 1.35 & $1.02 \sim 1.79$ \\
Age & 0.01 & 0.01 & 3.03 & 0.043 & 1.01 & $0.99 \sim 1.03$ \\
Carotid plaque & 0.37 & 0.21 & 2.30 & 0.014 & 1.45 & $0.95-2.21$ \\
Alanine aminotransferase (U/L) & 0.02 & 0.01 & 17.81 & 0.000 & 1.02 & $1.01 \sim 1.03$ \\
Aspartate aminotransferase (U/L) & 0.03 & 0.01 & 9.31 & 0.002 & 1.95 & $1.90 \sim 2.00$ \\
Uric acid & 0.00 & 0.00 & 2.46 & 0.001 & 1.03 & $1.02 \sim 1.04$ \\
Body mass index (kg/m $\left.{ }^{2}\right)$ & 1.48 & 0.15 & 102.19 & 0.000 & 4.38 & $3.29 \sim 5.84$ \\
Fasting plasma glucose (mmol/L) & 0.83 & 0.28 & 8.53 & 0.003 & 2.29 & $1.31 \sim 3.40$ \\
Total cholesterol (mmol/L) & -0.14 & 0.19 & 0.52 & 0.040 & 2.78 & $2.54-3.72$ \\
Triglyceride (mmol/L) & 0.85 & 0.15 & 30.83 & 0.000 & 2.33 & $1.73 \sim 3.14$ \\
HDL-C & -0.27 & 0.20 & 1.90 & 0.168 & 0.76 & $0.52 \sim 1.12$ \\
LDL-C & 0.32 & 0.20 & 2.56 & 0.000 & 2.83 & $2.35 \sim 3.34$ \\
Systolic blood pressure (mm Hg) & 0.06 & 0.23 & 0.06 & 0.800 & 1.06 & $0.68 \sim 1.66$ \\
Diastolic blood pressure (mm Hg) & 0.55 & 0.20 & 7.56 & 0.006 & 1.73 & $1.17 \sim 2.56$ \\
\hline
\end{tabular}

Table 3 - Multivariate logistic regression analysis for nonalcoholic fatty liver disease (NAFLD) after adjusting effects of confounders.

\begin{tabular}{lcccccc}
\hline $\begin{array}{l}\text { Multivariate logistic } \\
\text { regression analysis }\end{array}$ & B & S. E. & Wald test & $P$-value & Odds ratio & $\begin{array}{c}95 \% \\
\text { Confidence intervals }\end{array}$ \\
\hline Model 1 & 0.32 & 0.12 & 7.27 & 0.007 & 1.38 & $1.09 \sim 1.75$ \\
Model 2 & 0.28 & 0.12 & 5.40 & 0.020 & 1.33 & $1.05 \sim 1.68$ \\
Model 3 & 0.35 & 0.13 & 7.14 & 0.008 & 1.42 & $1.10 \sim 1.83$ \\
Model 4 & 0.30 & 0.14 & 4.38 & 0.036 & 1.35 & $1.02 \sim 1.79$ \\
\hline
\end{tabular}

Model 1 included only Helicobacter pylori infection. Age and carotid plaque status were added to Model 2. Model 3 was Model 2 with further adjustment for alanine aminotransferase, aspartate aminotransferase, and uric acid levels. Finally, more potential indicators and confounders, including fasting plasma glucose, total cholesterol, triglyceride, systolic blood pressure, diastolic blood pressure, low-density lipoprotein cholesterol, and body mass index were added to Model 4.

As presented in Figure 1, there was a positive correlation between $H$. pylori infection and TC $(p=0.015, \mathrm{r}=0.071$, Figure $2 \mathrm{~A}$ ). And $H$. pylori infection was also positively correlated with LDL-C ( $p=0.013, \mathrm{r}=0.072$, Figure 2B). Collectively, our results provide evidence that infection of $H$. pylori may be involved in the pathogenesis of NAFLD by influencing the metabolism of cholesterol.

Discussion. Nonalcoholic fatty liver disease is a frequent metabolic affecting nearly one-third of the world's population, but the underlying mechanism remains poorly understood. Many factors, namely, metabolic, behavioral, genetic, and environmental, may be responsible for NAFLD pathogenesis. ${ }^{18}$
Findings over recent years have established possible associations of $H$. pylori infection with NAFLD. ${ }^{19}$ Herein, a positive influence of $H$. pylori infection on NAFLD was demonstrated. Remarkably, after ruling out additional confounders, consisting of age, blood lipids, carotid plaque, blood glucose, liver enzymes, and blood pressure, $H$. pylori infection (95\% CI 1.02-1.79, OR $1.35, p=0.036$ ) remained the only independent factor for NAFLD. This finding is in accordance with that of a previous report which documented a positive correlation between NAFLD and $H$. pylori infection. ${ }^{20}$

The initial view of NAFLD is a hepatic indication of the metabolic disorder has been changing, and it is currently regarded that the occurrence of NAFLD may 
Table 4 - Effect of Helicobacter pylori (H. pylori) infection on metabolic syndrome indicators in nonalcoholic fatty liver disease (NAFLD) individuals.

\begin{tabular}{|c|c|c|c|c|}
\hline Biochemical profiles & $\begin{array}{c}\text { H. pylori }(+) \\
(\mathbf{n}=230)\end{array}$ & $\begin{array}{c}\text { H. pylori }(-) \\
(\mathbf{n}=299)\end{array}$ & $t / U$ & $P$-value \\
\hline Total cholesterol (mmol/L) & $5.24 \pm 1.10$ & $5.09 \pm 1.02$ & -2.47 & 0.014 \\
\hline Triglyceride (mmol/L) & $1.71 \pm 1.24$ & $1.69 \pm 1.34$ & -0.659 & 0.411 \\
\hline HDL-C & $1.31 \pm 0.37$ & $1.35 \pm 0.42$ & 1.71 & 0.087 \\
\hline LDL-C & $3.10 \pm 0.86$ & $2.98 \pm 0.82$ & -2.51 & 0.012 \\
\hline Systolic blood pressure (mm Hg) & $121.14 \pm 16.29$ & $120.86 \pm 16.79$ & -0.29 & 0.776 \\
\hline Diastolic blood pressure (mm Hg) & $78.85 \pm 11.74$ & $78.50 \pm 11.61$ & -0.50 & 0.614 \\
\hline Fasting plasma glucose (mmol/L) & $4.95 \pm 1.34$ & $4.89 \pm 1.48$ & -0.69 & 0.492 \\
\hline Body mass index $\left(\mathrm{kg} / \mathrm{m}^{2}\right)$ & $24.92 \pm 3.64$ & $24.59 \pm 3.89$ & -1.47 & 0.142 \\
\hline
\end{tabular}

HDL-C: high-density lipoprotein cholesterol, LDL-C: low-density lipoprotein cholesterol

A retrospective cohort study of Chinese asymptomatic adults

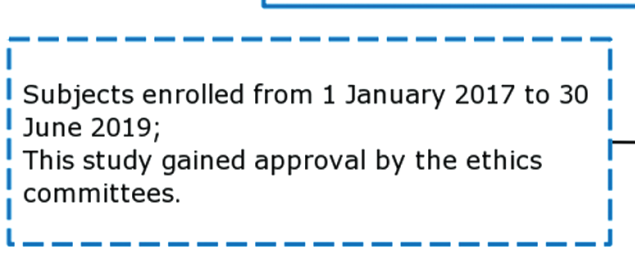

Subjects included $(n=1185 ;$ male:778, female:407)

(a) Subects who underwent health check-up;

(b) Subjects who simultaneous underwent abdominal color ultrasound, cervical color ultrasound, 13C-urea breath test (UBT), complete physical examination and had a complete set of biochemical tests.

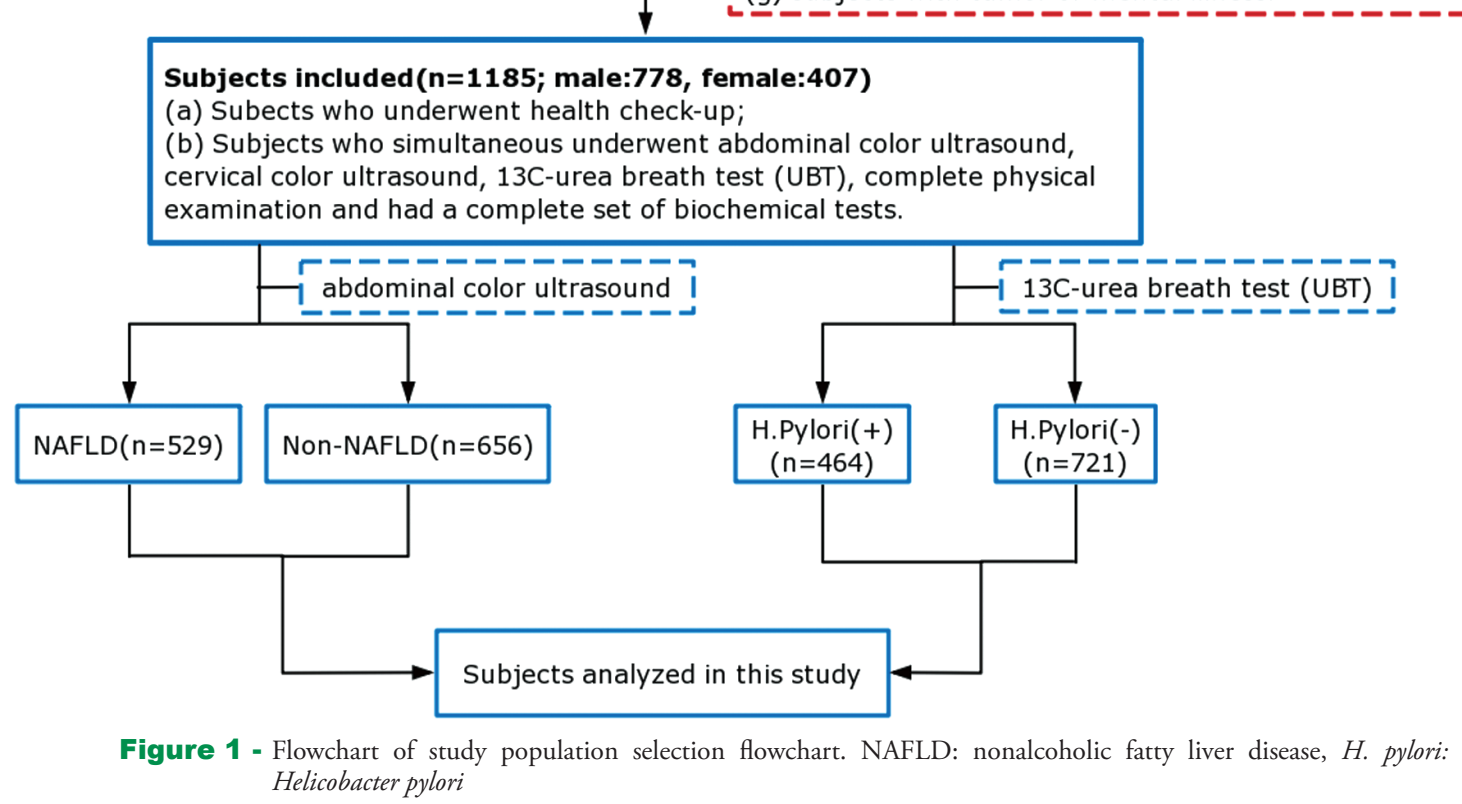

Figure 1 - Flowchart of study population selection flowchart. NAFLD: nonalcoholic fatty liver disease, H. pylori: Helicobacter pylori

Subjects excluded

( (a) a long history of heavy drinking: drinking alcohol at | least $210 \mathrm{~g}$ for male, $140 \mathrm{~g}$ for female per week in the past I I year;

I (b) previous treatment history of Methotrexate,

I amiodarone, glucocorticoid and tamoxifen;

(c) subjects with viral hepatitis, autoimmune hepatitis, hepatolenticular degeneration, total parenteral nutrition, lack of beta lipoprotein, congenital lipid atrophy, and celiac disease;

(d) taking proton pump inhibitors for 2 weeks, taking antibiotics and bismuth salts for 4 weeks before $13 \mathrm{C}$-urea i breath test;

I (e) a history of upper gastrointestinal;

I (f) a history of previous gastric surgery;

I (g) subjects with tumor or mental illness. 
A

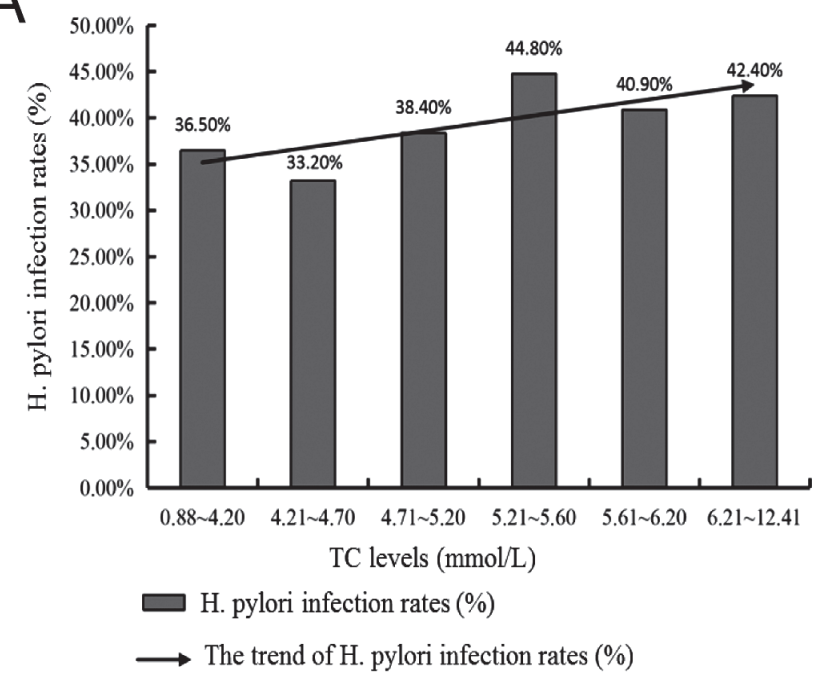

B

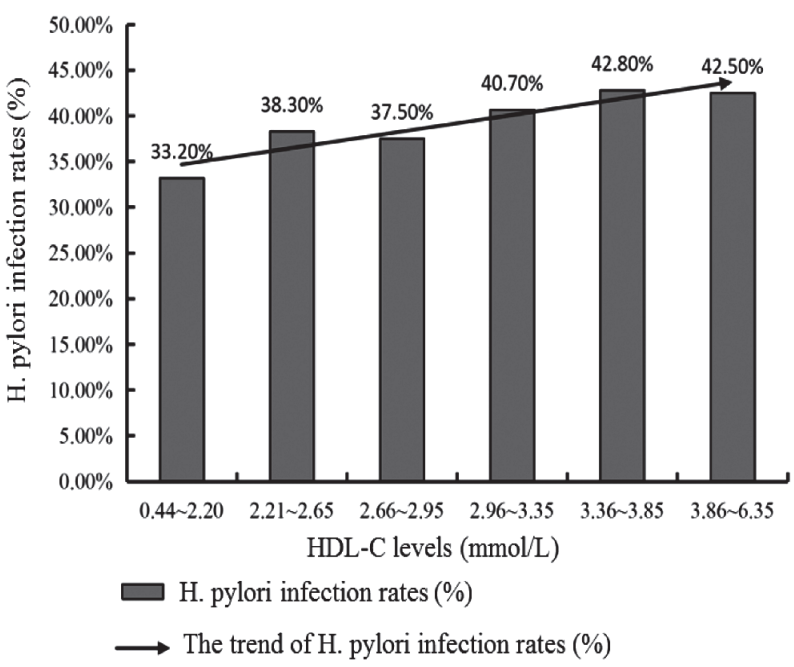

Figure 2 - Helicobacter pylori (H. pylori) infection rates in nonalcoholic fatty liver disease (NAFLD) individuals categorized by A) total cholesterol (TC) and B) low-density lipoprotein cholesterol (LDL-C).

precede metabolic syndrome. ${ }^{21}$ Thus, when combined with the analysis of several metabolic indices, we obtained higher weight, DBP, and SBP and elevated contents of TG, FPG, TC, BMI, and LDL-C (all $p<0.05)$ in individuals with NAFLD in contrast with those without NAFLD. We also identified DBP, BMI, FPG, and TG, as remarkable risk factors of NAFLD. Therefore, we speculated that a potential interaction exists between the NAFLD and metabolic syndrome constituents. As noted in another study, metabolic syndrome itself may contribute to the onset and progress of NAFLD, and vice versa. ${ }^{22}$

Abundant published research has demonstrated that infection of $H$. pylori is strongly associated with NAFLD, as well as metabolic syndrome. ${ }^{23-26}$ Herein, we also evaluated whether $H$. pylori infection affected various metabolic syndrome indicators (namely, HDL-C, SBP, FPG, TC, DBP, BMI, TG, and LDL-C). Notably, both LDL-C and TC contents were elevated in the $H$. pylori $(+)$ group $(p<0.05)$. Consequently, H. pylori infection may contribute to an escalation in LDL-C and TC contents. This finding is in accordance with a previous meta-analyses report that confirmed the influence of $H$. pylori infection on the lipid profile in serum. ${ }^{27}$ However, herein, the effects of $H$. pylori infection on other metabolic indicators were not verified. Here, we enumerate some possible reasons for our results. First, some guidelines before blood collection were provided to the participants. Some biochemical and physiological indicators of the participants in their original living environment (namely, kidney function, blood lipid, liver function, blood pressure, and blood glucose,) may have been underestimated. Second, the study included only subjects with higher education level working in government-affiliated institutions in Dali City. They are likely to have a higher level of health awareness than rural populations. Therefore, these study findings may be biased toward participants with better living standards, better dietary profiles, as well as good health awareness.

Study limitations. Our findings merit consideration, along with several potential limitations. First, this was a retrospective, single-center research. Another limitation of this study is the possible sample bias. Only subjects who underwent health check-ups were enrolled in the analysis. Thus, generalizing the findings to other populations is a challenge. Our present results require further verification using larger samples with different socioeconomic backgrounds. Third, no follow-up of the participants was performed. Fourth, we only confirmed that the infection of $H$. pylori was an independent risk factor for NAFLD in present study. The underlying mechanism of $H$. pylori infection evoking NAFLD was mere speculation. More in-depth prospective studies are required for the precise mechanistic association between H. pylori infection and NAFLD.

In summary, $H$. pylori infection may be a potential important contributor of NAFLD. However, further studies are needed to elucidate the precise mechanism of the relationship between $H$. pylori infection and NAFLD. 
Acknowledgment. This work was funded by Yunnan Health Training Project of High Level Talents (\#H-2019045), the Special Basic Cooperative Research Programs of Yunnan Provincial Undergraduate Universities' Association (\#202001BA070001-040 \& 202001BA070001-055), and the National Natural Science Foundation, China (\#81960363). We thank the Editage company in Shanghai (www.editage.cn) for their English language editing services of this manuscript.

\section{References}

1. Loomba R, Lim JK, Patton H, El-Serag HB. AGA Clinical practice update on screening and surveillance for hepatocellular carcinoma in patients with nonalcoholic fatty liver disease: Expert review. Gastroenterology 2020; 158: 1822-1830.

2. Zhou F, Zhou J, Wang W, Zhang XJ, Ji YX, Zhang P, et al. Unexpected Rapid Increase in the Burden of NAFLD in China from 2008 to 2018: A systematic review and meta-analysis. Hepatology 2019; 70: 1119-1133.

3. Estes C, Anstee QM, Arias-Loste MT, Bantel H, Bellentani S, Caballeria J, et al. Modeling NAFLD disease burden in China, France, Germany, Italy, Japan, Spain, United Kingdom, and United States for the period 2016-2030. J Hepatol 2018; 69: 896-904.

4. Younossi ZM. Non-alcoholic fatty liver disease - A global public health perspective. J Hepatol 2019; 70: 531-544.

5. Safari Z, Gerard P. The links between the gut microbiome and non-alcoholic fatty liver disease (NAFLD). Cell Mol Life Sci 2019; 76: 1541-1558.

6. Mansori K, Moradi Y, Naderpour S, Rashti R, Moghaddam $\mathrm{AB}$, Saed L, et al. Helicobacter pylori infection as a risk factor for diabetes: a meta-analysis of case-control studies. BMC Gastroenterol 2020; 20: 77.

7. Crowe SE. Helicobacter pylori Infection. N Engl J Med 2019; 380: 1158-1165.

8. Razuka-Ebela D, Giupponi B, Franceschi F. Helicobacter pylori and extragastric diseases. Helicobacter 2018; 23 Suppl 1: e12520.

9. Zhou BG, Yang HJ, Xu W, Wang K, Guo P, Ai YW. Association between Helicobacter pylori infection and nonalcoholic fatty liver disease: A systematic review and meta-analysis of observational studies. Helicobacter 2019; 24: e12576.

10. Yu YY, Cai JT, Song ZY, Tong YL, Wang JH. The associations among Helicobacter pylori infection, white blood cell count and nonalcoholic fatty liver disease in a large Chinese population. Medicine (Baltimore) 2018; 97: e13271.

11. Jiang T, Chen X, Xia C, Liu H, Yan H, Wang G, et al. Association between Helicobacter pylori infection and non-alcoholic fatty liver disease in North Chinese: a cross-sectional study. Sci Rep 2019; 9: 4874.

12. Okushin K, Takahashi Y, Yamamichi N, Shimamoto T, Enooku $\mathrm{K}$, Fujinaga $\mathrm{H}$, et al. Helicobacter pylori infection is not associated with fatty liver disease including non-alcoholic fatty liver disease: a large-scale cross-sectional study in Japan. BMC Gastroenterol 2015; 15: 25.

13. Baeg MK, Yoon SK, Ko SH, Noh YS, Lee IS, Choi MG. Helicobacter pylori infection is not associated with nonalcoholic fatty liver disease. World J Gastroenterol 2016; 22: 2592-2600.
14. Fan N, Peng L, Xia Z, Zhang L, Wang Y, Peng Y. Helicobacter pylori infection is not associated with non-alcoholic fatty liver disease: A cross-sectional study in china. front microbiol 2018; 9: 73 .

15. Cai O, Huang Z, Li M, Zhang C, Xi F, Tan S. Association between Helicobacter pylori infection and nonalcoholic fatty liver disease: A single-center clinical study. Gastroenterol Res Pract 2018; 2018: 8040262.

16. Zou B, Sun L, Wang X, Chen Z. The prevalence of single and multiple thyroid nodules and its association with metabolic diseases in Chinese: A cross-sectional study. Int J Endocrinol 2020; 2020: 5381012.

17. Xue Y, Zhou LY, Lu HP, Liu JZ. Recurrence of Helicobacter pylori infection: incidence and influential factors. Chin Med J (Engl) 2019; 132: 765-771.

18. Friedman SL, Neuschwander-Tetri BA, Rinella M, Sanyal AJ. Mechanisms of NAFLD development and therapeutic strategies. Nat Med 2018; 24: 908-922.

19. Okushin K, Tsutsumi T, Ikeuchi K, Kado A, Enooku K, Fujinaga $\mathrm{H}$, et al. Helicobacter pylori infection and liver diseases: Epidemiology and insights into pathogenesis. World J Gastroenterol 2018; 24: 3617-3625.

20. Liu R, Liu Q, He Y, Shi W, Xu Q, Yuan Q, et al. Association between Helicobacter pylori infection and nonalcoholic fatty liver: A meta-analysis. Medicine (Baltimore) 2019; 98: e17781.

21. Neves C, Rodrigues T, Sereno J, Simoes C, Castelhano J, Goncalves J, et al. Dietary Glycotoxins Impair Hepatic Lipidemic Profile in Diet-Induced Obese Rats Causing Hepatic Oxidative Stress and Insulin Resistance. Oxid Med Cell Longev 2019; 2019: 6362910.

22. Federico A, Zulli C, de Sio I, Del Prete A, Dallio M, Masarone $\mathrm{M}$, et al. Focus on emerging drugs for the treatment of patients with non-alcoholic fatty liver disease. World J Gastroenterol 2014; 20: 16841-16857.

23. Chen C, Zhang C, Wang X, Zhang F, Zhang Z, Ma P, et al. Helicobacter pylori infection may increase the severity of nonalcoholic fatty liver disease via promoting liver function damage, glycometabolism, lipid metabolism, inflammatory reaction and metabolic syndrome. Eur J Gastroenterol Hepatol 2020; 32: 857-866.

24. Alvarez CS, Florio AA, Butt J, Rivera-Andrade A, Kroker-Lobos MF, Waterboer T, et al. Associations between Helicobacter pylori with nonalcoholic fatty liver disease and other metabolic conditions in Guatemala. Helicobacter 2020: e12756.

25. Lim SH, Kim N, Kwon JW, Kim SE, Baik GH, Lee JY, et al. Positive association between Helicobacter pylori infection and metabolic syndrome in a Korean population: A multicenter nationwide study. Dig Dis Sci 2019; 64: 2219-2230.

26. Liou JM, Chen CC, Chang CM, Fang YJ, Bair MJ, Chen PY, et al. Long-term changes of gut microbiota, antibiotic resistance, and metabolic parameters after Helicobacter pylori eradication: a multicentre, open-label, randomised trial. Lancet Infect Dis 2019; 19: 1109-1120.

27. Shimamoto T, Yamamichi N, Gondo K, Takahashi Y, Takeuchi $\mathrm{C}$, Wada R, et al. The association of Helicobacter pylori infection with serum lipid profiles: An evaluation based on a combination of meta-analysis and a propensity score-based observational approach. PLoS One 2020; 15: e0234433. 